\title{
Antifilarial Activity of Eucalyptus globulus Labill. Leaves Against Brugia malayi
}

\author{
Vijai Lakshmi $^{1}$ and Shailja-Misra Bhattacharya ${ }^{2}$ \\ ${ }^{1}$ Division of Medicinal and Process Chemistry, CSIR-Central Drug Research Institute, Lucknow- 226001, U.P, India \\ ${ }^{2}$ Division of parasitology, CSIR-Central Drug Research Institute, Lucknow- 226001, U.P, India
}

Received: June 08, 2015; Accepted: December 08, 2015; Published (Web): February 17, 2016

\begin{abstract}
The present study is aimed to evaluate the antifilarial activity of Eucalyptus globules Labill. (Myrtaceae) against human lymphatic filarial parasite Brugia malayi in vitro and in vivo. The ethanolic extract of the leaves was tested in vitro on adult worms and microfilariae $(\mathrm{mf})$ of $B$. malayi and the active sample was further evaluated in vivo in B. malayi intraperitoneally (i.p.) transplanted in the jird model (Meriones unguiculatus) and Mastomys coucha subcutaneously infected with infective larvae (L3). The ethanolic extract of the leaves of the E. globulus was tested in vitro on adult worms and microfilariae ( $\mathrm{mf}$ ) of $B$. malayi and the active sample was further evaluated in vivo in B. malayi. The ethanolic extract was active in vitro (IC50: adult $=62.5 \mu \mathrm{g} / \mathrm{ml} ; \mathrm{mf}=31.2 . \mu \mathrm{g} / \mathrm{ml}$ ) where it demonstrated $66.7 \%$ adulticidal and embryostatic effect on $B$. malayi in Mastomys at a dose of $5 \times 100 \mathrm{mg} / \mathrm{kg}$ by oral route. The antifilarial test conducted was at $5 \times 100$ $\mathrm{mg} / \mathrm{kg}$ by subcutaneous route revealed excellent adulticidal efficacy resulting in to the death of $66.7 \%$ transplanted adult $B$. malayi in the peritoneal cavity of jirds in addition to noticeable microfilaricidal action on the day of autopsy. The findings revealed that the extract from the leaves of E. globulus contains promising in vitro and in vivo antifilarial activity against human lymphatic filarial parasite $B$. malayi which may be further explored to new antifilarial agents.
\end{abstract}

Key words: E. globulus, antifilarial activity, in vitro, in vivo, B. malayi

\section{Introduction}

Over the past few years, the interest in natural medicine has been increasing in industrialized societies because of the ever growing problem of side effects and high cost of synthetic medicines. Eucalyptus is a native genus from Australia belonging to Myrtaceae family and comprises about 900 species (Booker et al., 2004). More than 300 species of this genus contain volatile oils in their leaves. Some 20 species are known for their high content of 1,8-cineole (more than 70\%) which have been commercially used for the production of essential oils in pharmaceutical and cosmetic industries (Pino et al., 2002). In Tunisian folk medicine, inhalation of essential oil Eucalyptus sp. has traditionally been used to treat respiratory tract disorders such as pharyngitis, bronchitis, and sinusitis (Boukef, 1986). Consequently, the scientific interest in this field has been expanding. Researchers have demonstrated some efficacy of E. globulus essential oil against Haemophilus influenzae and Stenotrophomonas maltophilla (Cermelli et al.; 2008 et al. and Fabio et al., 2007). Few studies have reported the antiviral activity of Eucalyptus essential oils against Adenovirus, mumps and herpes simplex viruses (Schnitzler et al., 2001).

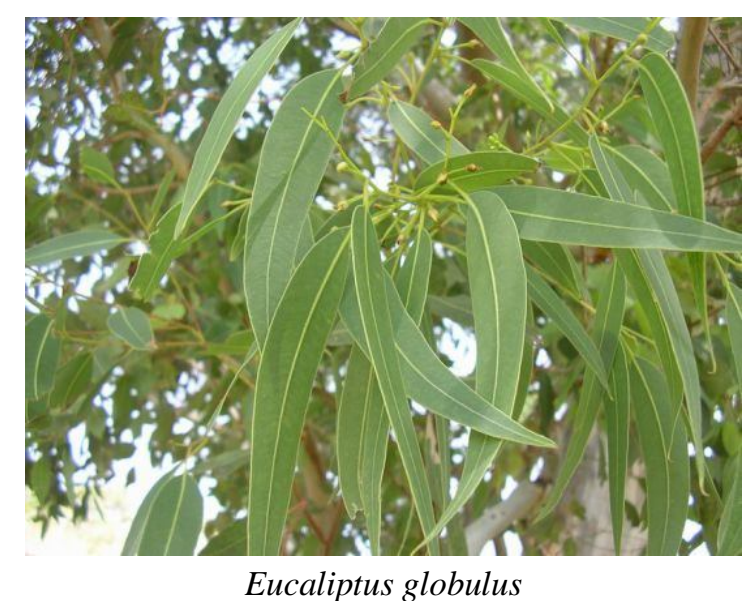

Correspondence to: Vijai Lakshmi; Phone: + 91-(0522)- 2254604; E-mail: vijlakshmius@yahoo.com 


\section{Materials and Methods}

Plant material: Leaves of the E. globulus (500g.) were purchased from the local market of Uttar Pradesh,, India and was authenticated at the Botany Department of the Central Drug Research Institute, Lucknow, India, where voucher specimen have been preserved with the code number 2191.

Extraction: The air dried leaves of the E. globulus $(500 \mathrm{~g})$ were powdered and percolated in $95 \%$ ethanol at room temperature for 24 hours, filtered and the process was repeated four times. All the extracts were mixed and filtered. Mixed ethanolic extract was concentrated under reduced pressure below $50{ }^{\circ} \mathrm{C}$ in a rotavapour to a green viscous mass, which was dried under high vacuum for 2 hours to remove the last traces of the solvent. Weight of the dried ethanolic extract $18.5 \mathrm{~g}$ which was used for the screening of antifilarial activity against $B$. malayi .

\section{Antifilarial activity: in vitro assays}

Sample preparation: $1 \mathrm{mM}$ stock solution of the ethanol extract of the E. globulus was prepared in dimethylsulfoxide.

Parasite isolation: The live adult $B$. malayi worms were isolated from the peritoneal cavity of jird (Meriones unguiculatus) infected 100-150 days earlier by intraperitoneal inoculation of 150-200 infective larvae (L3) of B. malayi recovered from experimentally infected mosquitoes, Aedes aegypti (McCall et al. 1973 After isolating the adult parasites, the peritoneal washing was passed through a membrane filter (pore size $5.0 \mathrm{~mm}$ ) and the microfilariae were pelleted by centrifugation (Singh et al., 1985). All the animals and experimental procedures were duly approved by the Animal Ethics Committee of CDRI, duly constituted under the provisions of the Committee for the Purpose of Control and Supervision on Experiments on Animals, Government of India. This study bears the approval no. IAEC/2011/120/Renew 01/dated 14/08/2012.

Primary in vitro screening: The actively motile female worms were placed individually wells of 48 well culture plate containing RPMI 1640 medium fortified with antibiotics (penicillin 100 units/mL, streptomycin sulfate $100 \mathrm{mg} / \mathrm{mL}$, and neomycin mixture; Sigma,
USA). Each well contained one female worm in $1 \mathrm{~mL}$ of the medium. Simultaneously, 10 microfilariae were suspended in $200 \mathrm{~mL}$ medium in each well of a 96 well culture plate (NUNC). The parasites were incubated at $37{ }^{\circ} \mathrm{C}$ in $5 \% \mathrm{CO}_{2}$ in air for 5 days in the presence of 10 $\mathrm{mM}$ concentration of the ethanol extract of leaves and the motility of parasites was monitored microscopically at regular time intervals. At the end of the experiment, adult parasites were transferred to fresh drug free medium for one hour at $37{ }^{\circ} \mathrm{C}$ to observe reversal, if any, in the worm motility. The worms were later processed individually for MTT [3-(4,5dimethylthiazol- 2-yl)-2,5-diphenyltetrazolium bromide] dye reduction assay as published earlier (Mukherjee et al., 1988) for checking their metabolic viability. The experiments were carried out in duplicate and the degree of loss in the motility as well as percent inhibition in MTT reduction in treated parasites over the untreated controls was assessed. The extract which consistently demonstrated their lethal effects on the parasites at $10 \mathrm{mM}$ (highest concentration tested) with $50 \%$ inhibition in MTT reduction as compared to untreated respective controls were considered as active extract (Mukherjee et al., 1988) while those bringing about $100 \%$ irreversible inhibition in motility of microfilariae were considered microfilaricidal.

(iv) Evaluation of $I C_{50}$ and $C C_{50}$ : The test samples found active in primary in vitro screen were followed for $\mathrm{IC}_{50}$ using four serial two fold dilutions of each sample starting from MIC in the same way as mentioned above. $\mathrm{IC}_{50}$ values were determined by Excel based line graphic template after plotting concentration values of each sample versus percent motility inhibition of parasite on $\mathrm{x}$ - and $\mathrm{y}$-axis. In vitro $\mathrm{CC}_{50}$ assay on Verocells (monkey kidney cell line) was performed as mentioned earlier (Misra et al. 2011). In brief, Vero cells (104/well/100 ml) in 96 well plate were exposed to seven three-fold serial dilutions of active test samples starting from $100 \mathrm{mM}$ at $37^{\circ} \mathrm{C}$ in a $\mathrm{CO}_{2}$ incubator. After $72 \mathrm{~h}$, resazurin dye was added and the plate was re-incubated for 3-4 h. The reaction was monitored fluorometrically at an excitation wavelength of $536 \mathrm{~nm}$ and emission at $588 \mathrm{~nm}$ in a fluorometric plate reader.

Scoring and activity evaluation criterion: The motility of the adult worms and microfilariae was 
scored as $0 \%$ motility reduction $(4 \mathrm{p}) ; 1-49 \%$ motility reduction (3p); 50-74\% motility reduction (2p); 75-99\% motility reduction $(1 \mathrm{p})$ and $100 \%$ motility reduction (dead) (Misra et al. 2011).

Determination of selectivity index (SI): The safety of the active samples was determined by assessing SI values $\left(\mathrm{CC}_{50} / \mathrm{IC}_{50}\right)$ The extract with $\mathrm{SI}$ values of 10 were considered safe and therefore further followed in vivo.

\section{In vivo antifilarial activity}

In vivo screening model: Intraperitoneal (i.p) transplantations of 10 females and five male adult worms of B. malayi were carried out in 6-8 week old male jirds. The jirds were anaesthetized by ketamine (50 mg/kg, i.p), animals were quickly shaved and a small incision was made on latero-ventral region of abdomen to introduce worms into the peritoneal cavity. The success of transplantation was affirmed by the presence of live microfilaria in a drop of peritoneal fluid aspired on day 4 and these jirds were selected to screen in vivo hits.

Treatment schedule: The ethanol extract of the leaves of E. globulus and the standard drug, diethylcarbamazine were administered subcutaneously at a dose of $100 \mathrm{mg} / \mathrm{kg}$ for five consecutive days to seven groups of transplanted jirds and each group had three animals. Three jirds received vehicle only and served as control group.

Assessment of antifilarial activity in the primary jird model: The treatment was initiated from day 5 post worm transplantation and the observations were continued till 45 days. On $45^{\text {th }}$ day, the jirds were euthanized along with the untreated controls to recover worms by peritoneal washings. The recovered parasites were counted and examined for motility, death or calcification. Live female worms were teased in a drop of phosphate buffer saline (PBS, $\mathrm{pH}$ 7.2) to dissect out the uterus for observing the uterine contents microscopically to assess the embryostatic effect of test samples, if any (Bajpai et al., 2005). The peritoneal washing collected on autopsy was microscopically observed to assess the effect of test sample on released microfilariae.
Statistical analysis: The statistical analysis of the data was carried out by PRISM 3.0 using one way ANNOVA (nonparametric). Dunnett's multiple (Bajpai et al., 2005) comparison test was applied to assess the statistical significance of the values between treated and control group. Values were expressed as mean $\pm \mathrm{SE}$. P $<0.05$ was considered as of low significance $(*)$ while $\mathrm{P}<0.01 / 0.001$ were considered as highly significant $(* * / * * *)$.

\section{Results}

The ethanolic extract of the leaves of E. globulus was tested in vitro on adult worms and microfilariae (mf) of B. malayi and the active sample was further evaluated in vivo in $B$. malayi intraperitoneally transplanted in the jird model and Mastomys coucha subcutaneously infected with infective larvae (L3). The ethanolic extract was found to be active in vitro $\left(\mathrm{IC}_{50}\right.$ : adult $=62.5 \mu \mathrm{g} / \mathrm{ml} ; \mathrm{mf}=31.2 . \mu \mathrm{g} / \mathrm{ml})$ and demonstrated $66.7 \%$ adulticidal and embryostatic effect on B. malayi, respectively, in Mastomys at a dose of $5 \times 100 \mathrm{mg} / \mathrm{kg}$. The antifilarial activity at $5 \times 100 \mathrm{mg} / \mathrm{kg}$ by subcutaneous route revealed excellent adulticidal efficacy resulting in the death of $66.7 \%$ transplanted adult $B$. malayi in the peritoneal cavity of jirds in addition to noticeable microfilaricidal action on the day of autopsy.

\section{Conclusion}

The present findings revealed that the ethanol extract of the leaves of E. globulus contains promising in vitro and in vivo antifilarial activity against human lymphatic filarial parasite $B$. malayi which can be further explored to isolate and characterize the active molecules to provide new antifilarial agents.

\section{Acknowledgments}

The authors are grateful to the Director of CSIR (Central Drug Research Institute), Lucknow, India for providing necessary research facilities to carry out the above work and also excellent library facility. The one of us (VL) emeritus scientist grateful to acknowledge HRDG, CSIR, New Delhi for providing financial support which helped in compiling this work for publication. 


\section{References}

Bajpai, P., Verma, S.K., Katiyar, D., Tewari, N., Tripathi R.P., Bansal, I., Saxena, J.K. and Bhattacharya, S.M. 2005. Search for new prototypes for the chemotherapy of filariasis: a chemotherapeutic and biochemical approach. Parasitol. Res. 95, 383-390.

Boukef, M.K. 1986. Médicine traditionnelle et pharmacopée, les plantes dans la médicine traditionnelle tunisienne. Agence de Coopération Culturelle et Technique 92, 9028-085.

Brooker, M.I. and Keing, D.A. 2004. Field guide to Eucalyptus (2nd ed.). In Bloomings Book. Melbourne, Northern Australia.

Cermelli, C., Fabio, A., Fabio, G. and Quaglio, P. 2008. Effect of ealyptus essential oil on respiratory bacteria and viruses. Curr. Microbiol. 56, 89-92.

Fabio, A., Cermelli, C., Fabio, G., Nicoletti, P. and Quaglio, P. 2007. Screening of the antibacterial effects of a variety of essential oils on microorganisms responsible for respiratory infections. Phytother. Res. 21, 374-377.

McCall, J.W., Malove, J.B., Hyong-Sun A. and Thompson, P.E. 1973. Mongolian jirds (Meriones unguiculatus) infected with Brugia malayi by the intraperitoneal route: a rich source of developing larvae adult filariae and microfilariae, J. Parasitol. 59, 436.
Misra, S., Verma, M., Mishra, S.K., Srivastava, S., Lakshmi, V. and Misra-Bhattacharya, S., 2011. Gedunin and Photogedunin of Xylocarpus granatum possess antifilarial activity against human lymphatic filarial parasite Brugia malayi in experimental rodent host, Parasitol. Res. 109, 1351-1360.

Mukherjee, M., Misra, S. and Chatterjee, R.K., 1988. Development of in vitro screening system for assessment of antifilarial activity of compounds, Acta Trop. 70, 251-255.

Pino, J.A., Marbot, R., Quert, R. and Garcia, H. 2002. Study of essential oils of Eucalyptus resinifera Smith, E. tereticornis Smith and Corymbia maculata (Hook.) Hill, K.D. \& Johnson, L.A.S. Grown in Cuba. J. Flavour Fragr. 17, 1-14.

Schnitzler, P., Schon, K. and Reichling, J. 2001.Antiviral activity of Australian tea tree oil and eucalyptus oil against herpes simplex virus in cell culture. Pharmazie 56, 343-347.

Singh, D.P., Rathore, S., Misra, S., Chatterjee, R.K., Ghatak, S. and Sen, A.B. 1985. Studies on causation of adverse reactions following diethylcarbamazine therapy (Dipetalonema viteae) in Mastomys natalensis, Trop. Med. Parasitol. 36, 21-24. 